\title{
Early bleb leak after trabeculectomy and prognosis for bleb failure
}

Abstract

Aim To determine whether early bleb leak after MMC trabeculectomy affects intermediate intraocular pressure (IOP) outcome.

Methods Retrospective case note review. All cases of MMC trabeculectomy with at least 1-year follow-up were included. Cases where a bleb leak occurred within the first month were identified. All cases without an early bleb leak formed the control group. Patient demographics and clinical factors were analysed to determine any factors predisposing to bleb leakage. IOPs were compared for 1 year postoperatively and final follow-up.

Results A total of 119 trabeculectomies were included. Of these $27(22.7 \%)$ had an early bleb leak. The remaining 92 cases formed the control group. Mean age of cases was 70.7 years. Mean follow-up time was 19.5 months. Mean time of detection of the bleb leak was 9 days (range 121 days). Four cases (14.8\%) were managed by primary resuturing. Thirteen cases $(48.1 \%)$ were managed conservatively with a bandage contact lens. Ten cases $(37.0 \%)$ resolved with expectant management. There was no statistically significant difference between the two groups with regards to IOP measurement at any time point. Intervention rates were similar with regards to bleb massage, 5-fluorouracil injection, and needling revision. No factors were identified between the two groups that predisposed to bleb leaks occurring. Conclusion Our data suggest that early bleb leak is not a poor prognostic indicator for intermediate bleb survival and IOP control in patients undergoing MMC trabeculectomy. No additional bleb manipulations compared with the control group were required to achieve a satisfactory IOP outcome.

Eye (2009) 23, 858-863; doi:10.1038/eye.2008.130; published online 23 May 2008
A Alwitry' ${ }^{1}$ A Rotchford², V Patel ${ }^{2}$, A. Abedin², J Moodie ${ }^{2}$ and AJ King ${ }^{2}$

Keywords: glaucoma; trabeculectomy; bleb leak

\section{Introduction}

Early postoperative bleb leak is a common complication of trabeculectomy and has significant risks associated with it including the risk of endophthalmitis or hypotony. ${ }^{1-13}$ The reported incidence of postoperative bleb leak after trabeculectomy ranges from 0 to $56 \%$. $^{1,14}$

As we increase efforts to achieve better longterm success with filtering surgery, antimetabolites have gained increasing popularity. Leaking blebs may be encountered in the early postoperative period, or months to years after filtering surgery. Early postoperative bleb leaks are most often related to surgical trauma to the conjunctiva or inadequate conjunctival closure and can be avoided by careful and meticulous surgical technique. Spontaneous late bleb leaks occur more frequently in glaucoma filtering surgery following adjunctive use of antimetabolites. ${ }^{15,16}$ These leaks may be uncomplicated and self-limiting or may be associated with sight-threatening complications. The plethora of treatment options for management of bleb leaks described in the literature ${ }^{17-22}$ reflects the widespread nature of this problem. It also reflects the failure of any one particular approach not only to resolve bleb leaks but also to prevent their recurrence.

The aim of this study is to present outcome data of trabeculectomy augmented with MMC and compare the postoperative course and late results of patients who had an early leak (within 1 month of surgery) compared with those with no leak. In addition we attempt to identify any factors that may predispose to the development of early bleb leaks.

\section{Methods}

All trabeculectomies were undertaken by an experienced glaucoma surgeon (AJK) or by a
No research funding. No proprietary interests. 
trainee surgeon under the direct supervision of the consultant between November 2000 and August 2004 were included in the study.

All the clinical records of patients who had had a trabeculectomy procedure during the study period were reviewed. Data on all cases were retrieved retrospectively from the medical case notes. Data collection was carried out by four of the authors (AA, AA, JM, and VP). Pre- and postoperative data were retrieved on the patients, including patient demographics, preoperative glaucoma status (visual fields parameters, intraocular pressures, medications), visual acuities, and postoperative progress. Patients with a follow-up of less than 12 months were excluded. Patients with a documented wound leak in the first postoperative month were included in group A (early bleb leak) whereas those with no early leak were included in group B (controls). Patients who had a bleb leak after 1 month were excluded from the study. All data collected were rechecked by one of the authors for accuracy against the patients' records (AJK).

Snellen visual acuities were converted to LogMar using a standardised chart (Journal of Cataract and Refractive Surgery).

\section{Operative procedure}

Anaesthetic was by either general anaesthesia or by a peribulbar technique. A Clark's lid speculum was placed and a $7 / 0$ silk corneal traction suture was placed for ocular stabilisation. For a fornix-based procedure, the incision was made in the conjunctiva from 11 to 1 o'clock at the limbus with wide blunt dissection to free tenons and conjunctiva from sclera posteriorly to a distance of approximately $8-10 \mathrm{~mm}$. For a limbus-based operation, a $10 \mathrm{~mm}$ incision was made through conjunctiva and tenon capsule approximately $10 \mathrm{~mm}$ from the limbus.

Conjunctiva and tenons were dissected anteriorly to the limbus being careful to avoid a conjunctival buttonhole, and posteriorly to enlarge the potential area of drainage and encourage posterior aqueous drainage.

With both techniques meticulous haemostasis was undertaken by monopolar diathermy. Cellulose sponges soaked in mitomycin-C at concentrations of $0.1-0.4 \mathrm{mg} / 1$ were then applied over the area of the proposed scleral flap and underneath the conjunctiva and tenons capsule as far posterior as possible for $1-4$ min depending upon preoperative risk factors for failure and per-operative assessment of the conjunctiva. Care was taken to avoid contact with the free edges of the conjunctiva. Copious irrigation with balanced salt solution was then applied to wash out any residual MMC solution.

A paracentesis was created with an MVR blade and an AC maintainer placed. A partial thickness $5 \times 4 \mathrm{~mm}$ rectangular trapezoid scleral flap was fashioned using a diamond blade initially and then a crescent blade. The anterior chamber was then entered with a keratome in the base of the scleral flap incision and a Kelly-Descemet punch was used to create a sclerectomy. An iridectomy was performed using Vannas scissors. The scleral flap was closed with two releasable 10/0 nylon sutures and one further releasable suture centrally to the posterior lip of the flap if drainage was assessed peroperatively to be excessive. Tension on the individual sutures was tailored to each individual case to allow slow egress of fluid from the AC beneath the edges of the sutured scleral flap.

For fornix-based procedures, the conjunctiva was closed with two $10 / 0$ nylon purse string sutures with or without placement of mattress sutures. For limbal-based procedures, the conjunctival incision was closed with a single running 10/0 vicryl suture. 'Water tightness' of wound closure was checked by the application of topical $2 \%$ fluorescein to the eye and insufflation of the anterior chamber with balanced salt solution. If a leak was noted this was managed with a further mattress suture if at the wound edge, or with a 10/0 nylon purse-string if it was a buttonhole away from the wound edge and insufflation and fluorescein application was repeated. This process was continued if necessary until no leak was detectable. If the paracentesis was leaking a single 10/0 nylon suture was placed.

A subconjunctival injection of $3 \mathrm{mg}$ Zinacef $\AA$ and $2 \mathrm{mg}$

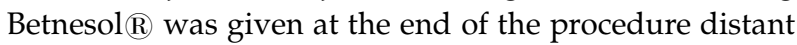
to the site of the trabeculectomy and a pad and patch was applied.

\section{Postoperative management}

All patients were treated with topical Maxidex six times daily and topical Voltarol four times daily for at least 3 months in addition to topical chloramphenicol $1 \%$ four times daily for the first 2 weeks. The steroid regimen was altered appropriately to encourage satisfactory bleb morphology.

Patients were reviewed on the first postoperative day and follow-up appointments were made for 1 week, 2 weeks, 3 weeks, 1 month, 2 months, 3 months, and 6 months postoperatively in the routine cases and at other times if deemed clinically necessary. Data were collected for the day 1 postoperative visit and for the 1 week, 1 month, 3 months, 6 month, and 1 year visits. Data were collected beyond this time point if it was available.

In all visits during the first postoperative month $2 \%$ fluorescein was applied over the bleb to detect any leakage if present. Postoperative bleb manipulation was undertaken in the form of bleb massage, removal of releasable sutures, subconjunctival injection of 5- 
fluorouracil (5-FU), and needling and 5-FU injection as clinically indicated.

If a bleb leak was noted one of three approaches was adopted. If there was a slow ooze or a leak that was precipitated by ocular pressure, expectant management was adopted. In cases where the bleb leak was significant but there was no hypotony or significant anterior chamber (AC) shallowing, a bandage contact lens was applied and if the leak was brisk or was associated with shallowing of the AC, choroidals or hypotony surgical repair of the leak was undertaken. In addition, oral acetazolamide was used in selected cases to reduce aqueous outflow and topical steroids were reduced or stopped when deemed necessary to promote wound healing. If a conservative approach failed to solve the bleb leak a more interventional approach was adopted.

\section{Statistics}

Definitions for success were defined at several IOP cutoff points. Success was classified as unqualified if IOP was achieved without any additional IOP-lowering drugs, and as qualified if additional medication was required to achieve the IOP. The distributions of binary variables were compared using the $\chi^{2}$-test. Means of continuous variables were compared by $t$-test when normally distributed, and Kruskal-Wallis test for nonparametric variables.

\section{Results}

Data were retrieved on 141 eyes of 119 patients. Second eye procedures were excluded from the analysis and thus data on 119 trabeculectomies were included for analysis.

Of the patients, 67 were male $(56.3 \%)$ whereas 52 were female $(43.7 \%)$ and their mean age was 70.7 years (SD 13.4 years; range $10-88$ years; median 74 years). Of the eyes, 60 were left eyes (50.4\%) whereas 59 were right eyes (49.6\%). Mean follow-up was 19.5 months (standard

Table 1 Aetiologies of glaucoma

\begin{tabular}{lc}
\hline & $n=119$ \\
\hline Inflammatory & $11(9.2 \%)$ \\
Primary open angle glaucoma & $73(61.3 \%)$ \\
Normal tension glaucoma & $15(12.6 \%)$ \\
Chronic angle closure glaucoma & $6(5.0 \%)$ \\
Pseudoexfoliative glaucoma & $3(2.5 \%)$ \\
Pigment dispersion syndrome & $3(2.5 \%)$ \\
Traumatic glaucoma & $3(2.5 \%)$ \\
Others ${ }^{\mathrm{a}}$ & $5(4.2 \%)$ \\
\hline${ }^{a}$ Ocular hypertension $=1, \quad$ congenital glaucoma $=1$, & plateau iris \\
syndrome $=1$, angle closure glaucoma $=2$. &
\end{tabular}

deviation 9.8 months; range 12-60 months; median 12 months).

Aetiology of glaucoma are shown in Table 1.

In 72 cases $(60.5 \%)$, there was no previous surgery of any sort; 91 cases $(76.4 \%)$ were phakic at the time of glaucoma surgery. Eight eyes (6.7\%) had had a laser peripheral iridotomy prior to surgery whereas seven (5.9\%) had had argon laser trabeculoplasty. Thirteen cases were repeat trabeculectomies (10.9\%). Early bleb leak was identified in 27 cases $(22.7 \%)$, who formed Group A; 92 cases $(77.3 \%)$ formed the control group (Group B). The demographic and preoperative characteristics of the leak and no-leak group are shown in Table 2.

Table 3 displays and statistically compares the pre- and postoperative intraocular pressure data between the two groups at each time point.

\section{Bleb leaks}

Of the 27 cases who had a bleb leak, the mean time of detection of the bleb leak was 9 days (range 1-21 days). Of all limbal-based procedures, $11.5 \%$ had a bleb leak whereas $25.8 \%$ of all fornix-based procedures had a bleb

Table 2 Demographic and preoperative characteristics of group A and group B

\begin{tabular}{lccc}
\hline & Group A & Group B & P-value \\
\hline Numbers (SD) & $27(22.7)$ & $92(77.3)$ & \\
Age (years) (SD) & $68.9(14.5)$ & $63.1(17.6)$ & 0.248 \\
Sex (M/F) & $17 / 10$ & $50 / 42$ & 0.430 \\
$\begin{array}{l}\text { Mean preoperative MD } \\
\text { (SD) }\end{array}$ & & & \\
Mean preoperative & $3.3(1.08)$ & $2.9(1.27)$ & 0.297 \\
drops (SD) & & & \\
Limbal/fornix & $3 / 24$ & $23 / 69$ & 0.185 \\
$\begin{array}{l}\text { Median preoperative } \\
\text { VA (range) }\end{array}$ & $0.2(0-3)$ & $0.2(0-3)$ & 0.29 \\
$\begin{array}{l}\text { Median postoperative } \\
\text { VA (range) }\end{array}$ & $0.18(-0.1-4)$ & $0.18(0-3)$ & 0.52 \\
\hline
\end{tabular}

Table 3 Pre- and post-operative intra-ocular pressures ( $\mathrm{mmHg}$ ) with $P$-values comparing groups $\mathrm{A}$ and $\mathrm{B}$

\begin{tabular}{lcrr}
\hline & Group A & Group B & P-value \\
\hline Pre-operative IOP (SD) & $25.08(7.23)$ & $25.05(7.85)$ & 0.984 \\
Day 1 IOP (SD) & $14.20(9.39)$ & $13.19(9.21)$ & 0.622 \\
Day 7 IOP (SD) & $12.20(8.35)$ & $9.96(8.57)$ & 0.233 \\
Month 1 IOP (SD) & $12.75(7.48)$ & $13.19(5.55)$ & 0.878 \\
Month 3 IOP (SD) & $11.37(5.30)$ & $10.41(4.30)$ & 0.350 \\
Month 6 IOP (SD) & $12.55(5.30)$ & $10.78(5.42)$ & 0.180 \\
Year 1 IOP (SD) & $12.98(5.64)$ & $12.00(3.66)$ & 0.397 \\
Year 2a IOP (SD) & $13.46(6.30)$ & $11.76(4.56)$ & 0.325 \\
${ }^{a} N=54$. & & &
\end{tabular}


leak $(P=0.19)$. Four cases $(14.8 \%)$ were managed by primary resuturing of the trabeculectomy bleb. Three leaks were at the conjunctival edge whereas one was related to a conjunctival buttonhole. All three of the leaks at the conjunctival edge occurred in fornix-based procedures.

Thirteen cases $(48.1 \%)$ were managed conservatively with a bandage contact lens with or without the use of oral acetazolamide. All of these cases had resolution of the leak within 1 week. Ten cases (37.0\%) resolved with expectant management.

There was no statistically significant difference between the two groups with regards concentration of MMC used $(P=0.67)$, the duration of application $(P=0.68)$, or the cumulative dose (concentration $\times$ duration; $P=0.64$ ).

\section{Interventions}

Table 4 compares numbers of interventions between the two groups.

Excluding interventions for the bleb leaks, the overall intervention rate was $77.2 \%$ in group $\mathrm{A}$ and $81.5 \%$ in group $\mathrm{B}\left(P=0.79, \chi^{2}\right)$. In group $\mathrm{B}$, seven cases $(7.6 \%)$ had an over-draining bleb leading to complications related to hypotony. In all cases over drainage occurred within the first postoperative month. In four of these cases AC was excessively shallow requiring return to theatre for reformation of $\mathrm{AC}$ with viscoelastic under topical anaesthesia. In two cases autologous blood was injected into the bleb (one of these cases required three injections). In the final case Palmberg sutures were placed 10 months postoperatively to limit an over-draining bleb.

Figure 1 shows IOP data plotted for each group against time and success rates as classified by various criteria shown in Table 5.

\section{Discussion}

Leakage from a trabeculectomy bleb is a potentially serious complication related to trabeculectomy surgery. An open communication between the anterior chamber and the ocular surface may predispose to infective complications including potentially blinding endophthalmitis. Consequent over drainage may result in a flat anterior chamber with the subsequent risks of hypotonous complications, including choroidal effusions, suprachoroidal haemorrhage, and hypotonous maculopathy. In addition, the resulting flat bleb may promote adhesion formation between the roof and the base of the bleb, thus detrimentally affecting bleb evolution and increasing the risk of bleb scarring and failure.

Early bleb leak (comprising wound leak and conjunctival button holes) may be related to deficient

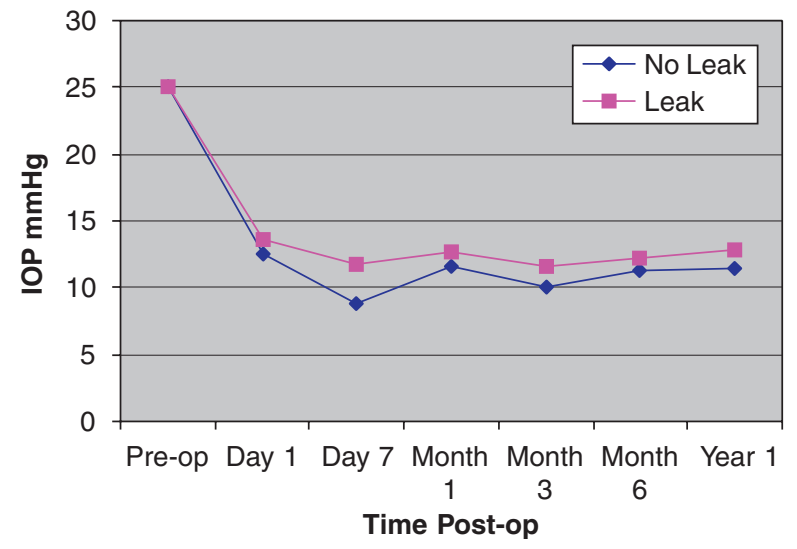

Figure 1 Intraocular pressure (IOP) data plotted for each group against time.

Table 5 Success of procedure with regards IOP reduction for specific IOP targets

\begin{tabular}{lccc}
\hline & Group A & Group B & P-value \\
\hline $\begin{array}{c}\text { Unqualified success } \\
<16 \mathrm{mmHg}\end{array}$ & $19(70.4 \%)$ & $60(65.2 \%)$ & 0.82 \\
$<18 \mathrm{mmHg}$ & $20(74.1 \%)$ & $66(71.7 \%)$ & 1.00 \\
$<21 \mathrm{mmHg}$ & $21(77.7 \%)$ & $66(71.7 \%)$ & 0.63 \\
Qualified success & & & \\
$<16 \mathrm{mmHg}$ & $21(77.8 \%)$ & $68(73.9 \%)$ & 0.80 \\
$<18 \mathrm{mmHg}$ & $23(85.2 \%)$ & $74(80.4 \%)$ & 0.78 \\
$<21 \mathrm{mmHg}$ & $26(96.3 \%)$ & $81(88.0 \%)$ & 0.29 \\
\hline${ }^{\mathrm{a}} \chi^{2}$-test. & & &
\end{tabular}

Table 4 Table statistically comparing numbers of intervention between groups

\begin{tabular}{|c|c|c|c|c|c|c|c|c|}
\hline & \multicolumn{2}{|c|}{$\begin{array}{l}\text { Massage (no. of patients } \\
\text { having intervention) }\end{array}$} & \multicolumn{2}{|c|}{$\begin{array}{l}\text { Suture removal (no. of patients } \\
\text { having intervention) }\end{array}$} & \multicolumn{2}{|c|}{$\begin{array}{l}\text { 5-FU injection (no. of patients } \\
\text { having intervention) }\end{array}$} & \multicolumn{2}{|c|}{$\begin{array}{c}\text { Needling }+5-F U \text { (no. of patients } \\
\text { having intervention) }\end{array}$} \\
\hline & Yes & No & Yes & No & Yes & No & Yes & No \\
\hline Group A & 11 & 16 & 12 & 15 & 10 & 17 & 7 & 20 \\
\hline Group B & 47 & 45 & 46 & 46 & 27 & 65 & 23 & 69 \\
\hline$P$-value & 0.34 & & 0.61 & & 0.45 & & 0.92 & \\
\hline
\end{tabular}


wound closure, patent stitch tracts, or surgical trauma to the conjunctiva. ${ }^{23}$ The use of augmenting antimetabolites may further increase the risk of bleb leak. ${ }^{24,25}$

This study showed bleb leakage to be considerably higher in fornix-based as opposed to limbal-based procedures with $25.8 \%$ of all fornix-based procedures having a bleb leak whereas $11.5 \%$ of all limbal-based procedures had a bleb leak, an observation that is supported by previous literature. ${ }^{1-5,26,27}$ Batterbury and Wishart ${ }^{1}$ reported postoperative leaks in $4 \%$ of all limbalbased procedures, whereas a significantly greater incidence was found in their fornix-based group (56\%).

Henderson $e a^{28}$ found an overall rate of bleb leak of $59 \% ; 65 \%$ occurred in fornix-based procedures compared with $24 \%$ in the limbal-based group. Mean time of leakage was 3.5 days. Their higher leak rate may be explained by the fact that their study was prospective and that the authors actively attempted to induce a leak by application of non-physiological pressure on the trabeculectomy bleb. The present study solely addressed clinically significant leaks in the physiological situation detected during normal clinical examination.

The present literature is conflicting with respect to the effect of bleb leak on long-term bleb function. The Fluorouracil Filtering Study (FFS) reported that early wound leak was related to poor final glaucoma surgery outcome. ${ }^{29}$ This is in contrast to the present study in which success appears unaffected by the presence of an early bleb leak. This difference may be explained to some degree by the fact that FFS addressed only limbal-based procedures and no mitomycin was used. In addition in the present study postoperative bleb intervention was undertaken to salvage failing blebs, this approach to postoperative manipulation was not commonly undertaken at the time of FFS.

In contrast several previous studies have suggested that limbal- and fornix-based flaps in trabeculectomy have similar outcomes..$^{2-5,27}$

One previous study directly assessed the question of bleb leak and the implications for final outcome. ${ }^{28}$ They found no difference between the failure rates of each group, however their follow-up time was only 6 months which is markedly shorter than the mean follow-up in this study which was 19.5 months. Despite this, and more stringent success criteria than those used in the aforementioned, the present study results are comparable with regards to success.

Their study also differed from our work in that they included numerous different procedures including triple procedures and 5-FU augmented trabeculectomies, whereas the present study concentrated solely on MMCaugmented trabeculectomy procedures. The use of this more potent antifibrotic agent may theoretically have implications for the incidence and clinical course of bleb leaks. It is possible that the use of MMC would inhibit fibroblastic activity in the subconjunctival/potential bleb space to such an extent that transient misdirection of liquid through the leak would not lead to irreversible flattening of the bleb as, when flow is subsequently restored to the appropriate potential bleb space on resolution of the leak, the subconjunctival space would have had the ability to still facilitate formation of an adequate drainage area.

In this study the mean time to leak detection was 9.5 days. This suggests that many of the bleb leaks develop shortly after surgery. It would therefore appear that despite the meticulous approach taken to wound closure at the time of surgery, early bleb leaks may still occur. This is likely due to changes in tissue swelling in the postoperative period, cutting through of sutures or changes in wound apposition caused by blinking or eye rubbing.

Bleb leaks in our study were not allowed to persist for more than approximately 2 weeks. It is possible that this protocol of relatively early surgical intervention to close a leak resulted in restoration of normal flow dynamics and good bleb evolution with consequent good function profile. This may have been helped by the use of MMC at the time of the original surgery, as this would have slowed down postoperative scarring and prolonged the period during which normal bleb function could be established.

When success was defined as an IOP of less than $18 \mathrm{mmHg}$ with or without medication, success was achieved in $85.2 \%$ of all bleb leak cases and $80.4 \%$ of the non-leak cases $(P=0.78)$.

All the surgeries were performed under the care of one surgeon and on a mainly Caucasian population, thus our results may not be applicable to patient populations consisting of other ethic backgrounds. There are several possible criticisms of this study. It is retrospective in nature and the precise clinical details of the bleb leak are absent from the patient's case notes, making meaningful qualification or quantification of bleb leakage difficult. It is unlikely that the leaks formed a homogenous group, apart from assumptions based upon the treatment applied at the time of leak discovery, it is difficult to determine the specific nature and morphology of the bleb leak. This may be important as it is reasonable to assume that a small leak at the conjunctival edge which did not significantly arrest flow into a formed trabeculectomy bleb would have markedly different consequences on bleb evolution when compared with a conjunctival buttonhole resulting in hypotony and a flat bleb. Precise clinical details of the exact nature and extent of the bleb leak were not accurately documented in the clinical notes. Further prospective studies should allow more precise qualification and quantification of bleb leaks and 
comparison of subgroups within the spectrum of early bleb leakage.

In conclusion it appears that in this study early bleb leakage, treated as described, was not associated with an adverse effect upon intermediate term bleb survival or IOP outcome after MMC augmented trabeculectomy. In addition it appears that no extra bleb manipulations had to be carried out in the leakage group to achieve this result.

\section{References}

1 Batterbury M, Wishart PK. Is high initial outflow of benefit in trabeculectomy? Eye 1993; 7: 109-112.

2 Shuster JN, Krupin T, Kolker AE, Becker B. Limbus- v fornix-based conjunctival flap in trabeculectomy. A longterm randomized study. Arch Ophthalmol 1984; 102: 361-362.

3 Tezel G, Kolker AE, Becker B. Comparative results of combined procedures for glaucoma and cataract: II. Limbus-based versus fornix-based conjunctival flaps. Ophthalmic Surg Lasers 1997; 28: 551-557.

4 Lemon LC, Shin DH, Kim C, Bendel RE, Hughes BA, Juzych MS. Limbus-based vs fornix-based conjunctival flap in combined glaucoma and cataract surgery with adjunctive mitomycin C. Am J Ophthalmol 1998; 125: 340-345.

5 Traverso CE, Tomey KF, Antonios S. Limbal- vs fornix-based conjunctival trabeculectomy flaps. Am J Ophthalmol 1987; 104: 28-32.

6 Matsuda T, Tanihara H, Hangai M, Chihara E, Honda Y. Surgical results and complications of trabeculectomy with intraoperative application of mitomycin C. Jpn J Ophthalmol 1996; 40: 526-532.

7 Hafetz L, Keren T, Naveh N. Early and late postoperative application of 5-fluorouracil following trabeculectomy in refractory glaucoma. Ophthalmic Surg 1994; 25: 715-719.

8 Ng PW, Yeung BY, Yick DW, Yu CB, Lam DS. Fornix-based trabeculectomy with Wise's suture technique in Chinese patients. Ophthalmology 2000; 107: 2310-2313.

9 Membrey WL, Poinoosawmy DP, Bunce C, Hitchings RA. Glaucoma surgery with or without adjunctive antiproliferatives in normal tension glaucoma: 1 intraoperative pressure control and complications. Br J Ophthalmol 2000; 84: 586-590.

10 Beatty S, Potamitis T, Kheterpal S, O'Neill EC. Trabeculectomy with augmented mitomycin $\mathrm{C}$ application under the scleral flap. Br J Ophthalmol 1998; 82: 397-430.

11 Manners TD. Phacotrabeculectomy with 'smile' incision, punch, and single releasable suture. Ophthalmic Surg Lasers 1998; 29: 570-574.

12 Parrish II RK, Schiffman JC, Feuer WJ, Heuer DK, Fluorouracil Filtering Surgery Study Group. Prognosis and risk factors for early postoperative wound leaks after trabeculectomy with and without 5-fluorouracil. Am J Ophthalmol 2001; 132: 633-640.
13 Edmunds B, Thompson JR, Salmon JF. The National Survey of Trabeculectomy. III. Early and late complications. Eye 2002; 16: 297-303.

14 Munden PM, Alward WL. Combined phacoemulsification, posterior chamber intraocular lens implantation, and trabeculectomy with mitomycin C. Am J Ophthalmol 1995; 119: 20-29.

15 Wilkins M, Indar A, Wormald R. Intra-operative mitomycin C for glaucoma surgery. Cochrane Database Syst Rev 2005; (4): CD002897.

16 Greenfield DS, Liebmann JM, Jee J, Ritch R. Late-onset bleb leaks after glaucoma filtering surgery. Arch Ophthalmol 1998; 116(4): 443-447.

17 Al-Shahwan S, Al-Torbak AA, Al-Jadaan I, Omran M, Edward DP. Long-term follow up of surgical repair of late bleb leaks after glaucoma filtering surgery. J Glaucoma 2006; 15(5): 432-436.

18 Halkiadakis I, Lim P, Moroi SE. Surgical results of bleb revision with scleral patch graft for late-onset bleb complications. Ophthalmic Surg Lasers Imaging 2005; 36(1): 14-23.

19 Matsuo H, Tomidokoro A, Tomita G, Araie M. Topical application of autologous serum for the treatment of lateonset aqueous oozing or point-leak through filtering bleb. Eye 2005; 19(1): 23-28.

20 Feldman RM, Altaher G. Management of late-onset bleb leaks. Curr Opin Ophthalmol 2004; 15(2): 151-154.

21 Tannenbaum DP, Hoffman D, Greaney MJ, Caprioli J. Outcomes of bleb excision and conjunctival advancement for leaking or hypotonous eyes after glaucoma filtering surgery. Br J Ophthalmol 2004; 88(1): 99-103.

22 Kee C, Hwang JM. Amniotic membrane graft for late-onset glaucoma filtering leaks. Am J Ophthalmol 2002; 133(6): 834-835.

23 Rand Allingham R (ed). Shields' Textbook of Glaucoma, 5th ed. Lippincott Williams \& Wilkins: Philadelphia, 2005.

24 Cohen JS, Greff LJ, Novack GD, Wind BE. A placebocontrolled, double-masked evaluation of mitomycin $\mathrm{C}$ in combined glaucoma and cataract procedures. Ophthalmology 1996; 103(11): 1934-1942.

25 DeBry PW, Perkins TW, Heatley G, Kaufman P, Brumback LC. Incidence of late-onset bleb-related complications following trabeculectomy with mitomycin. Arch Ophthalmol 2002; 120: 297-300.

26 Austin MW, Wishart PK. Reformation of the anterior chamber following trabeculectomy. Ophthalmic Surg 1993; 24: 461-466.

27 Alwitry A, Patel V, King AJ. Fornix vs limbal-based trabeculectomy. Eye 2005; 19(6): 631-636.

28 Henderson HW, Ezra E, Murdoch IE. Early postoperative trabeculectomy leakage: incidence, time course, severity, and impact on surgical outcome. Br J Ophthalmol 2004; 88(5): 626-629.

29 Filtering Surgery Study Group. Five-year follow-up of the Fluorouracil Filtering Surgery Study. Am J Ophthalmol 1996; 121(4): 349-366. 